SUSX-TH-95/71

hep-ph/9503457

March 1995

\title{
Bound States and Instabilities of Vortices
}

\author{
Michael Goodband*and Mark Hindmarsh ${ }^{\dagger}$ \\ School of Mathematical and Physical Sciences \\ University of Sussex \\ Brighton BN1 9QH \\ $U K$
}

\begin{abstract}
We examine the spectrum of small perturbations around global and local (gauge) abelian vortices, using simple numerical matrix techniques. The results are of interest both for cosmic strings and for their condensed matter analogues, superfluid and superconductor vortices. We tabulate the instabilities of higher winding number vortices, and find several bound states. These localised coherent oscillations of the order parameter can be thought of as particle states trapped in the core of the string.
\end{abstract}

*e-mail: m.goodband@sussex.ac.uk

${ }^{\dagger}$ e-mail: m.b.hindmarsh@sussex.ac.uk 


\section{Introduction}

The ideas of gauge unification and spontaneous symmetry breaking lie at the heart of modern particle physics. The well established view is of some Grand Unified Theory with gauge symmetry group $G$, suffering a series of spontaneous symmetry breakings which reduce the group to that of the Standard Model. In a cosmological context this series of spontaneous symmetry breakings is seen to occur as the universe cools. The first symmetry breaking occurs at an energy of the order $10^{16} \mathrm{Gev}$, and it is possible that topological defects may form, via the Kibble mechanism, at this transition [1, 2]. Such objects have many important implications: for example, defects of dimension 2 (domain walls) and 0 (monopoles) would come to dominate the energy density of the Universe soon after a GUT phase transition, which would necessitate a period of inflation if the theory admitted such defects.

When a $\mathrm{U}(1)$ symmetry is broken at a phase transition, there arises a network of cosmic strings [3], defects of dimension 1, which may provide seeds for large scale structure formation in the universe. Because strings are very thin and the cosmological scales of interest are many orders of magnitude bigger, they have been approximated by line sources with energy-momentum density $\operatorname{diag}(\mu, 0,0,-\mu)$ where $\mu$ is the energy density of the classical solution. There are, however, a few instances when one is interested in the string structure. They include: calculating scattering cross-sections (which are important for baryon number violation by strings [4]); finding bound states; quantum corrections to the energy; and curvature corrections for highly curved string [5]. All of these require a knowledge of the perturbation spectrum about the string.

In this paper we find the perturbation spectra in the backgrounds of global and local U(1) vortices. Simple U(1) strings provide an ideal test-bed for developing numerical techniques for eigenvalues. They are not trivial, like the kink [6], nor are they as geometrically involved as 3D objects such as the sphaleron [7]. However, one has to deal with all the same problems: gauge choice and numerical stability being the principal ones. Furthermore, they are a stepping stone on the way to an efficient technique for mapping the stability region of the electroweak string, which we shall describe in a separate publication [8]. Our methods are quite straightforward: we find the vortex solution using shooting or relaxation algorithms. The numerical profile functions are then used in constructing a discretised matrix of second derivatives of the fields. This matrix is then diagonalised to find the eigenvalues and eigenfunctions, using the standard linear algebra packages incorporated into MATLAB.

We find a continuum of scattering states, oscillatory at infinity, for each particle species, obeying the expected dispersion relation $\omega^{2}=\mathbf{k}^{2}+m^{2}$. There are unstable modes for higher winding number strings, corresponding to strings splitting in various ways. We tabulate these, and show how different angular momenta in the modes lead to different splitting configurations. We also find bound states: $N$ on global strings of winding number $N$ (we searched up to $N=5$ ); but on the gauge strings we found only one, which crossed into the continuum for a sufficiently large ratio of the scalar to gauge 
coupling. The bound states are excitations of the fields at the core of the string, decaying exponentially at infinity: one can think of them as particles trapped on the string.

As is well known, global strings are closely related to superfluid vortices, and local ones to flux tubes in superconductors. We conclude by discussing the connection and outlining the possible significance of our results for condensed matter physics.

\section{Perturbations about global strings}

The model considered in this section has a complex scalar field with the lagrangian density

$$
\mathcal{L}=\left(\partial^{\mu} \phi\right)^{*}\left(\partial_{\mu} \phi\right)-\lambda\left(\phi^{*} \phi-\eta^{2} / 2\right)^{2},
$$

invariant under global U(1) transformations. The field acquires a vacuum expectation value, breaking the symmetry and giving rise to a particle spectrum of one Goldstone boson and a Higgs boson of mass $\sqrt{2 \lambda \eta^{2}}$.

As is well known, there exist time independent classical vortex solutions to the equation of motion

$$
-\partial^{2} \phi+2 \lambda\left(|\phi|^{2}-\eta^{2} / 2\right) \phi=0 .
$$

The solutions are $z$-independent and are of the form $\phi=(\eta / \sqrt{ } 2) f(\rho) e^{i N \theta}$ where $N$ is the winding number. The energy per unit length of such configurations is given by

$$
E=\pi \eta^{2} \int \rho d \rho\left(\left(\frac{d f}{d \rho}\right)^{2}+\frac{N^{2}}{\rho^{2}} f^{2}+\frac{\lambda \eta^{2}}{2}\left(f^{2}-1\right)^{2}\right) .
$$

The energy is logarithmically divergent for large $\rho$

$$
E=E_{\text {core }}+\pi \eta^{2} N^{2} \ln \left(\frac{R}{\rho_{c}}\right),
$$

where $\rho_{c}$ is the core radius. In physical situations there exists a physical cut off, such as a container or a nearby anti-vortex, making the energy finite. It can be seen from the $N^{2} \ln (R)$ behaviour that two $N=1$ global strings have significantly lower energy than one $N=2$ global string. The decay of an $N=2$ string to two $N=1$ strings conserves the topological charge and so it would be expected that the decay would occur. Similarly, one expects the decay of higher $N$ strings into strings of lower winding number, provided the decay conserves the topological charge. Decays which would appear to be possible on energetic grounds (eg. one $N=2$ string to one $N=1$ string) but do not conserve the topological charge, do not occur because there is an infinite energy barrier seperating the two configurations. So only the $N=1$ string is expected to be stable.

Now consider $z$-independent perturbations to the classical string background $\phi_{c}(x)$ of the form $\phi_{c}\left(x^{i}\right)+\epsilon \delta \phi\left(x^{i}\right) e^{-i \omega t}$ and $\phi_{c}^{*}\left(x^{i}\right)+\epsilon \delta \phi^{*}\left(x^{i}\right) e^{i \omega t}$ where $i=1,2$. This gives corrections to the energy

$$
E=E\left(\phi_{c}\right)+\epsilon^{2} E_{2}+O\left(\epsilon^{3}\right)
$$


where

$$
E_{2}=\int d^{2} x\left(\delta \phi^{*}, \delta \phi\right) M\left(\begin{array}{c}
\delta \phi \\
\delta \phi^{*}
\end{array}\right)
$$

The matrix $M$ is the perturbation operator, obtained by taking second functional derivatives of the energy. To find the perturbative modes about the string, which can be interpreted as particle states, we solve the coupled eigenvalue problem

$$
M\left(\begin{array}{c}
\delta \phi \\
\delta \phi^{*}
\end{array}\right)=\omega^{2}\left(\begin{array}{c}
\delta \phi \\
\delta \phi^{*}
\end{array}\right)
$$

where

$$
M=\left(\begin{array}{cc}
-\nabla^{2}+2 \lambda\left(2\left|\phi_{c}\right|^{2}-\eta^{2} / 2\right) & 2 \lambda \phi_{c}^{* 2} \\
2 \lambda \phi_{c}^{2} & -\nabla^{2}+2 \lambda\left(2\left|\phi_{c}\right|^{2}-\eta^{2} / 2\right)
\end{array}\right) .
$$

The string is a cylindrically symmetric solution, so it is convenient to expand the perturbations in angular momentum states

$$
\delta \phi=\sum_{m} s_{m} e^{i(N+m) \theta} \quad \text { and } \quad \delta \phi^{*}=\sum_{-m} s_{-m}^{*} e^{-i(N-m) \theta} .
$$

It is also useful to rescale the coordinates by defining $r=\sqrt{2 \lambda \eta^{2}} \rho$ to remove the parameters $\lambda$ and $\eta$ from the equations. So rescaling and substituting in the string solution and perturbation expansion gives the coupled eigenvalue equations for the functions $s_{m}$ and $s_{-m}^{*}$

$$
\begin{aligned}
& \left(-\nabla_{r}^{2}+\frac{(m-N)^{2}}{r^{2}}+\frac{1}{2}\left(2 f^{2}-1\right)\right) s_{-m}^{*}+\frac{1}{2} f^{2} s_{m}=\omega^{2} s_{-m}^{*} \\
& \left(-\nabla_{r}^{2}+\frac{(m+N)^{2}}{r^{2}}+\frac{1}{2}\left(2 f^{2}-1\right)\right) s_{m}+\frac{1}{2} f^{2} s_{-m}^{*}=\omega^{2} s_{m}
\end{aligned}
$$

where $\omega^{2}$ is now dimensionless. To recover the dimensionful values, one should multiply by $m_{H}^{2}=2 \lambda \eta^{2}$. Note that the eigenvalue equations are the same after complex conjugation and changing $m \rightarrow-m$, so we need consider only $m \geq 0$.

If we set $\left(s_{m}, s_{-m}^{*}\right)$ to real and imaginary parts, we can seperate the above eigenproblem into two separate eigenproblems with explicitly real fields

$$
\begin{aligned}
\left(\begin{array}{cc}
D_{1}+\frac{1}{2}\left(2 f^{2}-1\right) & \frac{1}{2} f^{2} \\
\frac{1}{2} f^{2} & D_{2}+\frac{1}{2}\left(2 f^{2}-1\right)
\end{array}\right)\left(\begin{array}{c}
s_{m}^{r} \\
s_{-m}^{r}
\end{array}\right) & =\omega^{2}\left(\begin{array}{c}
s_{m}^{r} \\
s_{-m}^{r}
\end{array}\right) \\
\left(\begin{array}{cc}
D_{1}+\frac{1}{2}\left(2 f^{2}-1\right) & \frac{1}{2} f^{2} \\
\frac{1}{2} f^{2} & D_{2}+\frac{1}{2}\left(2 f^{2}-1\right)
\end{array}\right)\left(\begin{array}{c}
s_{m}^{i} \\
-s_{-m}^{i}
\end{array}\right) & =\omega^{2}\left(\begin{array}{c}
s_{m}^{i} \\
-s_{-m}^{i}
\end{array}\right)
\end{aligned}
$$

where $D_{1}=-\partial_{r}^{2}+(m-N)^{2} / r^{2}$ and $D_{2}=-\partial_{r}^{2}+(m+N)^{2} / r^{2}$. 
First consider the vacuum where $f=1$ and $N=0$. Then we have $D_{1}=D_{2}$ and we can diagonalise $M$ with the with the eigenvectors $(1,1)^{T} s_{m}$ and $(1,-1)^{T} s_{m}$ to give

$$
\begin{aligned}
\left(-\nabla_{r}^{2}+\frac{m^{2}}{r^{2}}+1\right) s_{0} & =\omega^{2} s_{0} \\
\left(-\nabla_{r}^{2}+\frac{m^{2}}{r^{2}}\right) s_{0} & =\omega^{2} s_{0}
\end{aligned}
$$

We can see that for the vacuum the solutions are Bessel's functions $J_{m}(k r)$ where for (3) $k$ is given by the continuum $\omega^{2}=k^{2}+1$ and for (4) by $\omega^{2}=k^{2}$. The eigenvalue spectrum of (3) corresponds to the spectrum a Higgs particle with rest mass 1 in units of $2 \lambda \eta^{2}$, whereas the eigenvalue spectrum from (4) has a continuum down to $\omega^{2}=0$ corresponding to Goldstone modes.

\section{Numerical method}

The eigenproblem above was solved with the boundary conditions $s_{m}, s_{-m} \rightarrow 0$ as $r \rightarrow \infty$ for various values of $m$. The profile for a string of winding $N$ was found by solving the equation for $f$ using a shooting method. This profile was then substituted into the discretized perturbation operator $M$ and the eigenvalue problem was then solved by the standard matrix methods used by MATLAB. This proceedure was performed for linearly discretized lattices with 64, 128 and 256 points. The results for the 256 point lattice are shown in Tables 1 and 2. The numerical values for the zero modes give an estimate of the accuracy of the method. The eigenvalue problem was also solved for a linear discretization in the variable $\rho$ where $\rho=\tanh (r)$. This gives a non-linear discretization in $r$ with more points in the core of the string. The results from this are in agreement with those quoted in Table 1 to the error given by the values for the zero modes.

\section{Zero modes}

The string is a solution centered at a point in a two dimensional plane and so there must be a perturbed field configuration corresponding to the infinitesimal translation

$$
\begin{aligned}
\phi(x) \rightarrow \phi(x+\delta x) & =\phi(x)+\frac{\partial \phi}{\partial x^{i}} \delta x^{i} \\
& =\phi+\epsilon \delta \phi
\end{aligned}
$$

For example, for the translation mode in the $x$-direction we take the $x$-derivative of the string solution

$$
\begin{aligned}
\frac{\partial \phi_{c}}{\partial x} & =\cos \theta \frac{\partial \phi_{c}}{\partial r}-\frac{\sin \theta}{r} \frac{\partial \phi_{c}}{\partial \theta} \\
& =\cos \theta \frac{d f}{d r} e^{i N \theta}-\frac{i N \sin \theta}{r} f e^{i N \theta}
\end{aligned}
$$




$$
=\frac{e^{i N \theta}}{2}\left(\left(\frac{d f}{d r}-\frac{N f}{r}\right) e^{i \theta}+\left(\frac{d f}{d r}+\frac{N f}{r}\right) e^{-i \theta}\right) .
$$

As can be seen, the translation mode has $m=1$, and the forms for $s_{1}, s_{-1}$ can be read off from above and shown to satisfy

$$
M\left(\begin{array}{c}
\delta \phi \\
\delta \phi^{*}
\end{array}\right)=0
$$

The numerical results for this zero mode are shown in the table and, as mentioned above, they give an estimate of the error in the eigenvalues.

\section{Bound states}

For the case $m=0$, we have $D_{1}=D_{2}$ and again we can diagonalise $M$ with the eigenvectors $(1,1)^{T} s_{0}$ and $(1,-1)^{T} s_{0}$ to give

$$
\begin{aligned}
& \left(-\nabla_{r}^{2}+\frac{N^{2}}{r^{2}}+\frac{1}{2}\left(3 f^{2}-1\right)\right) s_{0}=\omega^{2} s_{0} \\
& \left(-\nabla_{r}^{2}+\frac{N^{2}}{r^{2}}+\frac{1}{2}\left(f^{2}-1\right)\right) s_{0}=\omega^{2} s_{0} .
\end{aligned}
$$

The eigenvalue spectra of the above equations have continua as for the vacuum, plus the appearance of descrete eigenvalues with $\omega^{2}<1$ for (5) due to the potential confining states on the string. These later states can be interpreted as Higgs particles trapped on the string or as oscillations of the string thickness. The continuum states are scattering states with solutions tending to phase shifted Bessel's functions as $r \rightarrow \infty$. We found that there $N$ bound states for a string of winding number $N$ for $N=1, \ldots, 5$. The solutions to (6) consist solely of scattering states.

If we consider the perturbations as a function of $z$ then the perturbation expansion is

$$
\delta \phi=\sum_{m} s_{m} e^{i(N+m) \theta} e^{i k_{z} z} \quad \text { and } \quad \delta \phi^{*}=\sum_{-m} s_{-m}^{*} e^{-i(N-m) \theta} e^{-i k_{z} z} .
$$

and the solutions to the corresponding eigenvalue problem are similar to those given above but with $\omega^{2}=k_{r}^{2}+k_{z}^{2}+1$ etc. The bound modes given above can then be interpreted as travelling modes along the string. The profile for the bound mode of the $N=1$ vortex is shown in Figure 1 together with the string profile.

\section{Decay modes}

As noted earlier all global strings with $N \geq 2$ are expected to decay to strings of lower

winding number. The modes corresponding to these decays occur for $m \geq 2$. The eigenvalues are listed in Table 1, and we display $\phi+\epsilon \delta \phi$ (with a large $\epsilon$ ) in Figures 2-5, to give a graphical representaion of the decay. 
For $N=2$ there is an $m=2$ mode with negative eigenvalue, corresponding to the string splitting into two $N=1$ strings (Figure 2).

For $N=3$ there is an $m=2$ mode with negative eigenvalue corresponding to the string splitting into three $N=1$ strings in a line (Figure 3 ). The $m=3$ negative mode corresponds to the string splitting into three $N=1$ strings in a triangular configuration (Figure 4). There is also an $m=4$ negative mode which gives rise to five vortex cores (Figure 5). To see what is happening here consider the small $r$ behaviour of the perturbed string solution

$$
\begin{aligned}
\phi & =\phi_{c}+\delta \phi \\
\phi & =f e^{i 3 \theta}+s_{4} e^{i 7 \theta}+s_{-4} e^{-i \theta},
\end{aligned}
$$

which for small $r$ is given by

$$
\phi \sim r^{3} e^{i 3 \theta}+r^{7} e^{i 7 \theta}+r e^{-i \theta}
$$

so can see that $\phi \rightarrow r e^{-i \theta}$ as $r \rightarrow 0$. There is therefore the beginnings of an anti-vortex at the origin. Thus the net winding number is three, which is just as well since it is a conserved topological charge.

This is the first example of a more general feature that strings of winding number $N$ possess negative modes for $2 \leq m \leq 2 N-2$ where the string splits into strings of lower winding number, $m+1$ of them for $m>N$ and for $m=N$ the string splits into $N$ strings of unit winding. If we consider the energy of the string and the conservation of topological charge, the general rule for the allowed decays is that an $N$ wound string $\rightarrow M$ strings of winding $M_{i}$, such that

$$
\begin{aligned}
\sum_{i} M_{i} & =N \\
\sum_{i} M_{i}^{2} & <N^{2}
\end{aligned}
$$

The first condition is the conservation of topological charge. The second condition comes from the $N^{2} \ln (R)$ dependence of the energy for large $R$. The $\sum_{i} M_{i}^{2}=N^{2}$ possibility is ruled out due to the dependence of the core energy on $N$. If the energy of the string is divided up as

$$
\begin{aligned}
E_{\text {Goldstone }} & =\pi \eta^{2} N^{2} \int f^{2} \frac{d r}{r} \\
E_{\text {Higgs }} & =\pi \eta^{2} \int r d r\left(\left(\frac{d f}{d r}\right)^{2}+\frac{1}{4}\left(f^{2}-1\right)^{2}\right)
\end{aligned}
$$

then $E_{\text {Higgs }}$ is finite whereas $E_{\text {Goldstone }}$ has the $\ln (R)$ dependence for large $R$. As can be seen from Table 1 , the sum of $E_{\text {Higgs }}$ for the $M$ strings is greater than that of for the string of winding $N$. 
Although there are decay modes which begin to generate anti-vortices at the core for small perturbations, do they remain as the string decays further? In any physical situation this is unlikely. This is because the decay modes with anti-vortices have a number of unit winding number vortices arranged arround them in a symmetrical arrangement (for instance the $m=6$ decay mode of the $N=5$ string has a unit winding number anti-vortex at the center and six unit winding number vortices arranged arround it in a hexagon), and any slight asymmetry will result in the anti-vortex being attracted to one of the vortices and annihilating.

\section{Perturbations about local strings}

In this section we are considering the abelian-Higgs model with the lagragian density

$$
\mathcal{L}=-\frac{1}{4} F_{\mu \nu} F^{\mu \nu}+\left(D_{\mu} \phi\right)^{*}\left(D^{\mu} \phi\right)-\lambda\left(|\phi|^{2}-\eta^{2} / 2\right)^{2}
$$

where

$$
\begin{aligned}
D_{\mu} & =\left(\partial_{\mu}+i e A_{\mu}\right) \phi \\
F_{\mu \nu} & =\partial_{\mu} A_{\nu}-\partial_{\nu} A_{\mu} .
\end{aligned}
$$

The lagrangian is invariant under local U(1) transformations. This symmetry is broken by the Higgs field acquiring a vacuum expectation value, and this gives a particle spectrum of a massive gauge boson of mass e $\eta$ and a massive Higgs boson of mass $\sqrt{2 \lambda \eta^{2}}$. As shown by Nielsen and Olesen [9] there exist time independent classical vortex solutions to the time independent field equations

$$
\begin{aligned}
D_{a} D_{a} \phi & =2 \lambda\left(|\phi|^{2}-\eta^{2} / 2\right) \phi \\
\partial_{a} F_{a b} & =-i e\left(\phi^{*} D_{a} \phi-\phi\left(D_{a} \phi\right)^{*}\right)
\end{aligned}
$$

where $a=1,2,3$. The cylindrically symmetric $z$-independent vortex solutions can be written in the form $\phi=(\eta / \sqrt{ } 2) f(\rho) e^{i N \theta}, A_{\theta}=-N a(\rho) / e \rho$ and $A_{\rho}=0$ where $N$ is the winding number of the string. The energy per unit length of the configuration is given by

$$
E=\pi \eta^{2} \int \rho d \rho\left(\left(\frac{d f}{d \rho}\right)^{2}+\frac{N^{2}}{\rho^{2}} f^{2}(1-a)^{2}+\frac{\beta}{2}\left(f^{2}-1\right)^{2}+\frac{N^{2}}{2 \rho^{2}}\left(\frac{d a}{d \rho}\right)^{2}\right)
$$

where the new dimensionless coordinates $r$ is defined by

$$
r=\frac{e \eta}{\sqrt{2}} \rho=\frac{m_{A}}{\sqrt{2}} \rho,
$$

and the parameter $\beta$ is

$$
\beta=\frac{m_{H}^{2}}{m_{A}^{2}}=\frac{2 \lambda}{e^{2}}
$$


It will also be convenient to rescale the fields by making the replacements

$$
\phi \rightarrow \frac{\phi \eta}{\sqrt{2}}, \quad A_{\mu} \rightarrow \frac{\eta}{\sqrt{2}} A_{\mu} .
$$

Dimensionless fields and coordinates will be used throughout this section. The stability of $N>1$ Nielsen-Olesen vortices to splitting into vortices of lower winding has been studied previously [10], where it was shown that they are stable for $\beta<1$ and unstable for $\beta>1$. This can be seen from Figure 6 showing the energy per vortex for three values of $\beta$. For $\beta>1$ the energy per vortex is lowest for $N=1$, and so a vortex with winding number greater than one will split into vortices of lower winding number and so move left on the curve. For $\beta<1$ the reverse is true.

\section{Perturbations and gauge fixing}

Now, consider perturbations to the classical string solution of the form $\phi_{c}(x)+\epsilon \delta \phi(x) e^{-i \omega t}$, $\phi_{c}^{*}(x)+\epsilon \delta \phi^{*}(x) e^{i \omega t}$ and $A_{\mu}(x)+\epsilon \delta A_{\mu}(x) e^{-i \omega t}$. This gives corrections to the action

$$
S=S\left(\phi_{c}, A_{\theta}^{c}\right)+\epsilon^{2} S_{2}+O\left(\epsilon^{3}\right),
$$

where

$$
S_{2}=\frac{1}{2} \int d^{4} x \delta \Phi^{\dagger} \mathcal{D} \delta \Phi
$$

and where $\mathcal{D}$ is the perturbation operator and $\delta \Phi^{\dagger}=\left(\delta \phi^{*} e^{i \omega t}, \delta \phi e^{-i \omega t}, \delta A_{\mu} e^{-i \omega t}\right)$. The perturbation operator $\mathcal{D}$ is given by

$$
\begin{aligned}
\mathcal{D}= & \left(\begin{array}{ccc}
-\partial^{2}-2 i A_{\mu} \partial^{\mu}+A_{\mu}^{2}-\beta\left(2|\phi|^{2}-1\right) & -\beta \phi^{2} & -2 i \partial_{\mu} \phi-i \phi \partial_{\mu}+2 A_{\mu} \phi \\
-\beta \phi^{*^{2}} & -\partial^{2}+2 i A_{\mu} \partial^{\mu}+A_{\mu}^{2}-\beta\left(2|\phi|^{2}-1\right) & 2 i \partial_{\mu} \phi^{*}+i \phi^{*} \partial_{\mu}+2 A_{\mu} \phi^{*} \\
i \partial_{\mu} \phi^{*}-i \phi^{*} \partial_{\mu}+2 A_{\mu} \phi^{*} & -i \partial_{\mu} \phi+i \phi \partial_{\mu}+2 A_{\mu} \phi & g^{\mu \nu} \partial^{2}-\partial_{\mu} \partial_{\nu}+2 g^{\mu \nu}|\phi|^{2}
\end{array}\right) .
\end{aligned}
$$

and the equations of motion for the perturbations are $\mathcal{D} \delta \Phi=0$.

In order to set up an eigenvalue problem for the perturbations we need to remove the linear derivative terms, as well as removing the gauge degrees of freedom. To do this we choose the background gauge condition [11]

$$
F(A)=\partial_{\mu} \delta A^{\mu}-\left(\delta \phi^{*} \phi_{c}-\phi_{c}^{*} \delta \phi\right)=0 .
$$

which is imposed by adding the gauge fixing term

$$
\mathcal{L}_{G F}=\frac{1}{2}|F(A)|^{2}
$$

to the lagrangian. This enables us to seperate out the time derivatives to give the eigenvalue equations

$$
M^{G F}\left(\begin{array}{c}
\delta \phi \\
\delta \phi^{*} \\
\delta A_{i}
\end{array}\right)=\omega^{2}\left(\begin{array}{c}
\delta \phi \\
\delta \phi^{*} \\
\delta A_{i}
\end{array}\right)
$$


and

$$
\left(-\nabla^{2}+2|\phi|^{2}\right)\left(\begin{array}{l}
\delta A_{0} \\
\delta A_{z}
\end{array}\right)=\omega^{2}\left(\begin{array}{l}
\delta A_{0} \\
\delta A_{z}
\end{array}\right)
$$

where $i=1,2$ and the gauge fixed perturbation operator is

$$
\begin{aligned}
& M^{G F}= \\
& \quad\left(\begin{array}{ccc}
-\nabla^{2}-2 i A_{i} \nabla_{i}+A_{i}^{2}+\beta\left(2|\phi|^{2}-1\right)+|\phi|^{2} & (\beta-1) \phi^{2} & -2 i \nabla_{i} \phi+2 A_{i} \phi \\
(\beta-1) \phi^{*^{2}} & -\nabla^{2}+2 i A_{i} \nabla_{i}+A_{i}^{2}+\beta\left(2|\phi|^{2}-1\right)+|\phi|^{2} & 2 i \nabla_{i} \phi^{*}+2 A_{i} \phi^{*} \\
2 i \nabla_{i} \phi^{*}+2 A_{i} \phi^{*} & -2 i \nabla_{i} \phi+2 A_{i} \phi & -\delta^{i j} \nabla^{2}+2 \delta^{i j}|\phi|^{2}
\end{array}\right) .
\end{aligned}
$$

The $\delta A_{0}$ and $\delta A_{z}$ perturbations decouple because the background string solution is independent of $t$ and $z$.

The gauge condition (9) above does not fix the gauge completely. For comparison, consider the more familiar coulomb gauge $\nabla \cdot A=0$, where it is still possible to make gauge transformations

$$
A_{i} \rightarrow A_{i}-\nabla_{i} \Lambda
$$

and the gauge transformed field still satisfy the gauge, as long as $\Lambda$ is harmonic. Similarly for the background gauge condition it is still possible to make gauge transformations for $\Lambda$ satisfying

$$
\left(-\partial^{2}+2|\phi|^{2}\right) \Lambda=0
$$

This is why in quantum field theory the above gauge fixing term would be accompanied by a Fadeev-Popov ghost term of the form

$$
\mathcal{L}_{F P G}=\frac{1}{2} \Lambda^{*}\left(-\partial^{2}+2\left|\phi_{c}\right|^{2}\right) \Lambda
$$

In $2+1$ dimensions the perturbation operator for $\delta A_{0}$ and the ghosts is the same, so $\delta A_{0}$ and $\Lambda$ will have the same eigenvalue spectrum. In any calculation of quantum corrections, the perturbation operator for the ghosts enters with the opposite sign and a factor of 2 compared to that for $\delta A_{0}$. Thus the eigenvalue spectrum of the ghosts cancels that from $\delta A_{0}$ and a subset of the spectrum from $M^{G F}$. We can therefore ignore $\delta A_{0}$, and we must beware that not all the eigenvalues of $M^{G F}$ correspond to physical states.

In $3+1$ dimensions, where the modes can have a $z$ dependence, the ghosts cancel the contributions to the energy from a linear combinations of $\delta A_{0}$ and $\delta A_{z}$, and the spectrum built on the unphysical eigenmodes of $M^{G F}$.

\section{Numerical method}

We expand the scalar field perturbations in angular momentum states and the gauge field in total angular momentum states given by

$$
A_{+}=\frac{e^{-i \theta}}{\sqrt{2}}\left(A_{r}-i \frac{A_{\theta}}{r}\right) \quad \text { and } \quad A_{-}=\frac{e^{i \theta}}{\sqrt{2}}\left(A_{r}+i \frac{A_{\theta}}{r}\right)
$$


The background string solution is

$$
\phi_{c}=f e^{i N \theta}, \quad A_{+}^{c}=\frac{i N a}{\sqrt{2} r} e^{-i \theta}, \quad A_{-}^{c}=\frac{-i N a}{\sqrt{2} r} e^{i \theta},
$$

and the perturbations are

$$
\begin{aligned}
\delta \phi & =\sum_{m} s_{m} e^{i(N+m) \theta} & \delta \phi^{*} & =\sum_{m} s_{-m}^{*} e^{-i(N-m) \theta} \\
\delta A_{+} & =\sum_{m} i a_{m} e^{i(m-1) \theta} & \delta A_{-} & =-\sum_{m} i a_{-m}^{*} e^{i(m+1) \theta} .
\end{aligned}
$$

Substituting in the above gives the eigenvalue equations

$$
\left(\begin{array}{cccc}
D_{1} & V & A & B \\
V & D_{2} & B & A \\
A & B & D_{3} & 0 \\
B & A & 0 & D_{4}
\end{array}\right)\left(\begin{array}{c}
s_{m} \\
s_{-m}^{*} \\
a_{m} \\
a_{-m}^{*}
\end{array}\right)=\omega^{2}\left(\begin{array}{c}
s_{m} \\
s_{-m}^{*} \\
a_{m} \\
a_{-m}^{*}
\end{array}\right)
$$

where

$$
\begin{aligned}
D_{1} & =-\nabla^{2}+\frac{(N(1-a)+m)^{2}}{r^{2}}+\beta\left(2 f^{2}-1\right)+f^{2} \\
D_{2} & =-\nabla^{2}+\frac{(N(1-a)-m)^{2}}{r^{2}}+\beta\left(2 f^{2}-1\right)+f^{2} \\
D_{3} & =-\nabla^{2}+\frac{(m-1)^{2}}{r^{2}}+2 f^{2} \\
D_{4} & =-\nabla^{2}+\frac{(m+1)^{2}}{r^{2}}+2 f^{2} \\
V & =(\beta-1) f^{2} \\
A & =\sqrt{2}\left(\frac{d f}{d r}+\frac{N f}{r}(a-1)\right) \\
B & =-\sqrt{2}\left(\frac{d f}{d r}-\frac{N f}{r}(a-1)\right) .
\end{aligned}
$$

As before, the eigenvalue equations are the same after complex conjugation and changing $m \rightarrow-m$ so we need consider only $m \geq 0$.

If we resolve the functions $s_{m}, s_{-m}^{*}, a_{m}, a_{-m}^{*}$ into real and imaginary parts then, as for the global string, the complex eigenproblem separates into two eigenproblems with explicitly real fuctions

$$
\left(\begin{array}{cccc}
D_{1} & V & A & B \\
V & D_{2} & B & A \\
A & B & D_{3} & 0 \\
B & A & 0 & D_{4}
\end{array}\right)\left(\begin{array}{c}
s_{m}^{r} \\
s_{-m}^{r} \\
a_{m}^{r} \\
a_{-m}^{r}
\end{array}\right)=\omega^{2}\left(\begin{array}{c}
s_{m}^{r} \\
s_{-m}^{r} \\
a_{m}^{r} \\
a_{-m}^{r}
\end{array}\right)
$$




$$
\left(\begin{array}{cccc}
D_{1} & V & A & B \\
V & D_{2} & B & A \\
A & B & D_{3} & 0 \\
B & A & 0 & D_{4}
\end{array}\right)\left(\begin{array}{c}
s_{m}^{i} \\
-s_{-m}^{i} \\
a_{m}^{i} \\
-a_{-m}^{i}
\end{array}\right)=\omega^{2}\left(\begin{array}{c}
s_{m}^{i} \\
-s_{-m}^{i} \\
a_{m}^{i} \\
-a_{-m}^{i}
\end{array}\right)
$$

where $D_{\alpha}, V, A$ and $B$ are given above.

First, consider the vacuum, where $f=1, N=0$ and $A_{i}=0$. Then $A=B=0, D_{1}=$ $D_{2}$ in the above, and the upper left block can be diagonalised with the eigenvectors $(1,1)^{T} s_{m}$ and $(1,-1)^{T} s_{m}$ to give the four Bessel's equations

$$
\begin{aligned}
\left(-\nabla^{2}+\frac{m^{2}}{r^{2}}+2 \beta\right) s_{m} & =\omega^{2} s_{m} \\
\left(-\nabla^{2}+\frac{m^{2}}{r^{2}}+2\right) s_{m} & =\omega^{2} s_{m} \\
\left(-\nabla^{2}+\frac{(m-1)^{2}}{r^{2}}+2\right) a_{m} & =\omega^{2} a_{m} \\
\left(-\nabla^{2}+\frac{(m+1)^{2}}{r^{2}}+2\right) a_{-m} & =\omega^{2} a_{-m} .
\end{aligned}
$$

The eigenvalue spectra of these are $\omega^{2}=k^{2}+2 \beta$ for (12) corresponding to that of a Higgs particle, and $\omega^{2}=k^{2}+2$ for the other three corresponding respectively to the longitudinal and transverse states of the massive gauge boson.

In the presence of the string background we still obtain the continua given above, but as for the global string, there are discrete eigenvalues correponding to particle states trapped on the string. The profiles $f(r)$ and $a(r)$ were solved for with a relaxation method on the energy functional, and then substituted into the eigenvalue equations above. The eigenproblem was solved with the boundary conditions $s_{m}, s_{-m}, a_{m}, a_{-m} \rightarrow 0$ as $r \rightarrow \infty$ for various values of $m$, by the same method used for the global string. This was performed for linearly descretized lattices with 50 and 100 points. The results given in Table 3 are for 100 points and the translation modes give an estimate of the accuracy. Note that the error in the translation modes is greater for $\beta=4$ than $\beta=1$ or 0.25 . This is because the Higgs core is narrower with a much sharper transition from 0 to 1 than in the other cases, and so there are fewer points describing this region. One might think that a similar situation would hold for the gauge field for $\beta=0.25$ but its transition from 0 to 1 is more gentle.

\section{Zero modes}

After gauge fixing the only mode which can have zero eigenvalue is the translation mode. The field configurations corresponding to a gauge fixed infinitesimal translation are

$$
\phi(x) \rightarrow \phi(x+\delta x)=\phi(x)+\left(D_{j} \phi\right) \delta x^{j}
$$




$$
\begin{aligned}
& =\phi+\epsilon \delta \phi \\
A_{i}(x) \rightarrow A_{i}(x+\delta x) & =A_{i}(x)+F_{j i} \delta x^{j} \\
& =A_{i}+\epsilon \delta A_{i} .
\end{aligned}
$$

These perturbations satisfy the gauge condition (9) by the equations of motion. They also satisfy

$$
M \delta \Phi=0 .
$$

by the equations of motion. The numerical results for the translation eigenvalues of $M^{G F}$ are given in Table 3.

\section{Bound states}

Let us first consider $m=0$. In this case $D_{1}=D_{2}$ and $D_{3}=D_{4}$, and $M$ has eigenvectors of the form $\left(s_{0}, s_{0}, a_{0}, a_{0}\right)^{T},\left(s_{0},-s_{0}, a_{0},-a_{0}\right)^{T}$. Hence there are two sets of solutions for $s_{0}$ and $a_{0}$, each satisfying one of two equations:

$$
\begin{aligned}
& \left(\begin{array}{cc}
D_{1}+V & A+B \\
A+B & D_{3}
\end{array}\right)\left(\begin{array}{l}
s_{0} \\
a_{0}
\end{array}\right)=\omega^{2}\left(\begin{array}{l}
s_{0} \\
a_{0}
\end{array}\right) \\
& \left(\begin{array}{cc}
D_{1}-V & A-B \\
A-B & D_{3}
\end{array}\right)\left(\begin{array}{l}
s_{0} \\
a_{0}
\end{array}\right)=\omega^{2}\left(\begin{array}{l}
s_{0} \\
a_{0}
\end{array}\right) .
\end{aligned}
$$

For each $N$, we find that there exists a maximum value of $\beta$ below which equation (16) possesses one bound state solution, corresponding to some sort of coupled Higgs-gauge boson trapped on the string. For the $N=1$ string the bound state exists for $\beta<1.5$. For $\beta>1.5$ this bound mode becomes one of a number of modes in the continuum, for which the Higgs field is bound but the gauge field is not. These modes correspond to some sort of resonant scattering state of the gauge field. For $\beta<1$ there are similar resonant scattering modes, but with the Higgs and gauge fields interchanged. For higher values of $N$ there is still only one bound mode, but the upper limit on $\beta$ is higher. There are also several resonant scattering states.

The solutions to equation (17) also possess bound states for a range of $\beta$. For the range $1<\beta<8$ there is one such mode. For $\beta<1$, and for higher $N$, where there are a number of bound states. By examining the asymptotic form of the perturbation operator, one can establish that the mass squared is 2, and thus we are dealing with modes of the gauge field. The gauge mode is trapped on the string because it has a lower mass inside the Higgs core, so we would expect the bound mode to have lower energy and for there to be more of them when the Higgs core is wider than the gauge field core. This is indeed the case. However, as we warned earlier, gauge field modes are not necessarily physical. In fact, we find that these vector bound states have very similar eigenvalues to the ghost modes, strongly indicating that they are just a result of the residual gauge invariance in the background gauge. 
Table 3 shows the eigenvalues of the lowest bound modes occuring for equation (16), equation (17) and for the ghosts. One can see that the ghost bound modes eigenvalues are very close to those from equation (17). The zero mode eigenvalues give an indication of the error: the difference between the gauge field and the ghost eigenvalues is actually greater than the departure of the numerical zero mode eigenvalue, but we believe that our identification of them is nevertheless correct.

In $3+1$ dimensions, there is also the $A_{z}$ field to consider. This has exactly the same perturbation equation as the ghosts and $\delta A_{0}$. As there are in total 2 ghost degrees of freedom, they can only cancel one linear combination of $\delta A_{0}$ and $\delta A_{z}$, leaving the other for real physical propagating modes, at least one of which is a bound state for $\beta<8$. Thus we have a massive gauge boson confined to the string.

\section{Decay modes}

Abelian-Higgs strings with $N>1$ for $\beta>1$ are unstable to decay to strings of lower winding number. The gauge field present in the local strings has the effect of removing the energy's logarithmic dependence on the radius, and so the allowed decays of local strings are $N$ wound string $\rightarrow M$ strings of winding $M_{i}$ with $\sum_{i} M_{i}=N$ and $M_{i} \geq 1$. For $\beta<1$ the modes are the same but the eigenvalues become positive. Consider the eigenvalues given in Table 3 for the $m=2$ mode for $N=2$. When $\beta=4$ the string is unstable to splitting into two $N=1$ strings. As is well known $\beta=1$ strings do not interact and so $\omega^{2}=0$ for this case. Now when $\beta=0.25$ this splitting mode has a small positive eigenvalue. This mode then corresponds to two $N=1$ strings oscillating about their center of mass position. Again if we consider the $z$ direction we obtain travelling waves along the string. Table 3 gives the eigenvalues of the splitting modes for various $\beta$.

\section{Discussion}

For global strings of unit winding number, we have found a bound mode with zero angular momentum which, when quantised, corresponds to a bound Higgs particle. The interaction terms in the lagrangian, however, give a vertex for the Higgs boson to decay to two Goldstone bosons, with a decay rate $\Gamma \sim m_{H}$. A Higgs boson occurs as a resonance in the scattering of two Goldstone bosons at a centre of mass energy $m_{H}^{2}$. Similarly, the Higgs boson trapped on the string will be seen as a resonance at a centre of mass energy of approximately $0.81 m_{H}^{2}$. Indeed, in a strongly coupled theory, the state could only be seen as a very broad resonance, rather like the $\sigma$ in $\pi \pi$ scattering.

For local strings we have found a bound coupled Higgs-gauge boson for $\beta<1.5$ and a bound gauge boson in $3+1$ dimensions for $1<\beta<8$. There are vertices for a Higgs boson to decay to gauge bosons, but for the bound modes this cannot occur due to energy conservation. So for $U(1)$ cosmic strings, there are bound modes for the above $\beta$ ranges. Populations of these bound states could have interesting effects: if they are truly 
stable, they could alter the equation of state of the string, adding to the mass density and subtracting from the tension. However, in a realistic model it is rather unlikely that the string fields will not be coupled to light fields, which offer a decay route to the bound states.

Global strings possess a condensed matter analogue in the Ginzburg-Pitaevski theory 112, 13] of superfluid Helium II. In the G-P theory the free energy of the condensate is given by

$$
F=\int d^{3} x\left(\frac{\hbar^{2}}{2 m_{4}}|\nabla \psi|^{2}-\alpha|\psi|^{2}+\frac{\beta}{2}|\psi|^{4}\right),
$$

where $\psi$ is the condensate wave function, which for stationary superfluid has $\left|\psi_{0}\right|^{2}=$ $\rho_{s} / m_{4}=\alpha / \beta$, where $\rho_{s}$ is the superfluid density and $m_{4}$ is the mass of a helium atom. The equation of motion is first order in time, commensurate with the non-relativistic nature of the system:

$$
i \hbar \frac{\partial \psi}{\partial t}=\frac{\delta F}{\delta \psi^{*}}
$$

There exists an extremum of the free energy of the form $f(r) e^{i N \theta}$ where $f(r)$ is the same as in Section 1. This solution decribes a vortex in the superfluid in which the superfluid flows at velocity $v_{s}=\hbar N / m_{4} r$ about a line along the $z$-axis, where the superfluid density is zero. This is analogous to the global cosmic string. Unfortunately, we cannot rely too heavily on this description of vortices in Helium II, because the core size is of the same order as the inter-atomic spacing - the theory is strongly coupled. It is nevertheless interesting to expand about the vortex solution to find the spectrum of states in the vortex background. To make contact with the dimensionless version of the perturbation matrix $M$ of Section 2, it is convenient to scale all distances in units of the correlation length $\xi=\sqrt{ }\left(\hbar^{2} / 2 m_{4} \alpha\right)$, and to use a dimensionless field $\chi=\psi \sqrt{ }(\beta / \alpha)$. Then for perturbations $\delta \chi$ we find

$$
i \hbar \frac{\partial}{\partial t}\left(\begin{array}{c}
\delta \chi \\
\delta \chi^{*}
\end{array}\right)=\alpha\left(\left|\psi_{0}\right|^{2} \xi^{3}\right) M\left(\begin{array}{c}
\delta \chi \\
\delta \chi^{*}
\end{array}\right) .
$$

Thus the energy eigenvalues in the matrix background of states with momentum $\hbar k_{z} / \xi$ along the string are

$$
\varepsilon=\alpha\left(\left|\psi_{0}\right|^{2} \xi^{3}\right)\left(\omega^{2}+k_{z}^{2}\right) .
$$

Of particular interest is the spectrum of bound states at $\omega^{2} \simeq 0.81$, which we believe has not been convinvingly established before in Helium II vortices. These are not the "bound" states found by Pitaevski [14], which are modes with $m=1$ associated with the translation of the vortex as a whole. What we are discussing is a zero angular momentum oscillation of the size of the vortex - a "sausage" mode. Bound states at angular momentum 0 and 2 have been reported by Grant [15], but the accuracy of the numerics is open to question, for both states were found very close to threshold $\left(\omega^{2}=1\right)$. We find no evidence of bound states at $m=2$, and at $m=0 \omega^{2}$ is approximately $20 \%$ below threshold. 
These bound modes may be observable as a resonance in the scattering of phonons or second sound off the core of the vortex, but in the light of the comments above about the applicability of G-P theory to such a vortex, this may not be observable.

The Abelian-Higgs model also has a condensed matter analogue in the GinzburgLandau theory [16] of superconductivity. It has been shown that there is a range of conditions for which G-L theory is applicable [17].

In G-L theory the free energy is given by

$$
F=\int d^{3} x\left(\frac{\hbar^{2}}{2 m}\left|\left(\nabla_{a}-\frac{2 i e}{\hbar} A_{a}\right) \psi\right|^{2}+\frac{B_{a}^{2}}{2 \mu_{0}}-\alpha|\psi|^{2}+\frac{\beta}{2}|\psi|^{4}\right),
$$

where $\psi$ is condensate wave function and $B$ is the magnetic field. For the condensate in the absence of magnetic fields $\left|\psi_{0}\right|^{2}=\alpha / \beta$. There are two length scales to use in constructing a dimensionless free energy functional, the correlation length $\xi$, and the penetration depth $\Lambda=\sqrt{ }\left(\beta m / 4 e^{2} \mu_{0} \alpha\right)$. The Ginzburg-Landau parameter is defined as the ratio $\kappa=\Lambda / \xi$. Defining a dimensionless gauge field $v_{i}=2 e \Lambda A_{i} / \hbar$, we find

$$
F=\frac{1}{2 \kappa^{2}} \alpha\left(\left|\psi_{0}\right|^{2} \Lambda^{3}\right) \int d^{3} y\left(\left|\left(\nabla_{a}-i v_{a}\right) \chi\right|^{2}+\frac{1}{2}(\nabla \times \boldsymbol{v})^{2}-2 \kappa^{2}|\chi|^{2}+\kappa^{2}|\chi|^{4}\right) .
$$

We see that the Ginzburg-Landau parameter is analogous to $\sqrt{ }(\beta / 2)$, where $\beta$ is the ratio of scalar to gauge couplings used earlier. This gives the same perturbation matrix $M$ (and $M^{G F}$ ), up to an overall scale factor, and so the energy eigenvalues of the states in the superconductor vortex background are

$$
\varepsilon=\frac{1}{2 \kappa^{2}} \alpha\left(\left|\psi_{0}\right|^{2} \Lambda^{3}\right)\left(\omega^{2}+k_{z}^{2}\right)
$$

The bound modes on the string exist for a range of $\kappa$. Converting the $\beta$ limits into $\kappa$ gives that for $1 / \sqrt{2}<\kappa<\sqrt{3} / 2$ there are bound modes of type (a) (coupled Higgs-vector modes), while for $1 / \sqrt{2}<\kappa<2$ there are bound modes of type (b) (longitudinal gauge field modes).

Again, the observability of such modes is an open question, complicated by the fact that superconductor vortices are already known to have quasi-particle bound states [18.

\section{Acknowledgements}

We are grateful to Chris Jones and David Waxman for helpful conversations. This work was supported by PPARC: MG by studentship number 93300941 and MH by Advanced Fellowship number B/93/AF/1642.

\section{References}

[1] T.W.B. Kibble, J. Phys. A9, 1387 (1976) 
[2] A. Vilenkin and E.P.S. Shellard, Cosmic Strings and Other Topological Defects (Cambridge University Press, Cambridge, 1994)

[3] M.B. Hindmarsh and T.W.B. Kibble, preprint NI94025, IMPERIAL/TP/94-95/5, SUSX-TH-94-74, hep-ph/9411342 (1994), to appear in Rep. Prog. Phys.

[4] W. Perkins, L. Perivolaropoulos, A-C. Davis, R. Brandenberger and A. Matheson, Nucl. Phys. B353, 237 (1991)

[5] K. Maeda and N. Turok, Phys. Lett. 202B, 376 (1988); R. Gregory and P. Orland Nucl. Phys. B428, 221 (1994)

[6] R. Rajaraman, Solitons and Instantons (North Holland, Amsterdam, 1982)

[7] D. Diakonov, M. Polyakov, P. Sieber, J. Schaldach and K. Goeke, preprint, hep-ph/9502245 (1995)

[8] M. Goodband and M. Hindmarsh, in preparation.

[9] H.B. Nielsen and P. Olesen, Nucl. Phys. B61, 45 (1973)

[10] E.B. Bogomol'nyi, Sov. J. Nucl. Phys. 24, 449 (1976), [Yad. Phys. 24, 861 ]

[11] T.P. Cheng and L-F. Li, Gauge theory of elementary particle physics (Oxford University Press, Oxford, 1984)

[12] V.L. Ginzburg and L.P. Pitaevskii, Sov. Phys. J.E.T.P. 7, 858 (1958), [Zh. Eksp. Teor. Fiz. 34, 1240]

[13] D.R. Tilley and J. Tilley, Superfluidity and Superconductivity $2^{\text {nd }}$ ed. (Adam Hilger Ltd, Bristol and Boston, 1986)

[14] L.P. Pitaevski, Sov. Phys. J.E.T.P. 13, 451 (1961), [Zh. Eksp. Teor. Fiz. 40, 646]

[15] J. Grant, J. Phys. A4, 695 (1971)

[16] V.L. Ginzburg and L.D. Landau, Zh. Eksp. Teor. Fiz. 20, 1064 (1950)

[17] L.P. Gorkov, Sov. Phys. J.E.T.P. 9, 1364 (1959), [Zh. Eksp. Teor. Fiz. 36, 1918 ]

[18] C. Caroli, P. de Gennes and J. Matricon, Phys. Lett. 9, 307 (1963) 


\section{Table captions}

Table 1: $E_{\text {Higgs }}$ of global strings with winding number $N$ in units $\pi \eta^{2}$ and $m=0$ bound mode eigenvalues in units of $2 \lambda \eta^{2}$.

Table 2: Eigenvalues of decay modes of global strings of winding number $N$, in units of $2 \lambda \eta^{2} . m$ is the angular momentum of the mode and the $M_{i}$ values are the winding numbers of the strings formed by the decay.

Table 3: Eigenvalues for local string modes in units of $e^{2} \eta^{2} / 2$. The modes shown are a)Higgs-gauge boson bound mode, b)gauge boson bound mode, c)ghost bound mode t)translation mode, and s)splitting modes.

\section{Figure captions}

Figure 1: Global string field profile $f(r)$ for $N=1$ and the $m=0$ bound mode field profile $s_{0}(r)$.

Figure 2: $m=2$ decay mode for a $N=2$ global string.

Figure 3: $m=2$ decay mode for a $N=3$ global string.

Figure 4: $m=3$ decay mode for a $N=3$ global string.

Figure 5: $m=4$ decay mode for a $N=3$ global string.

Figure 6: The energy per vortex for $N=1, \ldots, 5$ local strings in units of $\pi \eta^{2}$. 


\begin{tabular}{|l|l|l|llllll|}
\hline $\mathrm{N}$ & $E_{\text {Higgs }}$ & zero mode & $\omega^{2}$ of $\mathrm{m}=0$ bound mode & & \\
\hline 1 & 0.7833 & 0.0012 & 0.8134 & & & & \\
2 & 2.4149 & 0.0019 & 0.6299 & 0.9554 & & & \\
3 & 4.9835 & 0.0013 & 0.5180 & 0.8747 & 0.9727 & & \\
4 & 8.5712 & 0.0013 & 0.4437 & 0.7926 & 0.9314 & 0.9822 & \\
5 & 13.0310 & 0.0014 & 0.3907 & 0.7206 & 0.8835 & 0.9527 & 0.9976 \\
\hline
\end{tabular}

Table 1:

\begin{tabular}{|l|l|l|r|ll|}
\hline $\mathrm{N}$ & $\mathrm{m}$ & $M_{i}$ & $\sum_{i} M_{i}^{2}$ & $\omega^{2}$ & \\
\hline 2 & 2 & 1,1 & 2 & -0.2093 & \\
3 & 2 & $1,1,1$ & 3 & -0.1283 & \\
& 3 & $1,1,1$ & 3 & -0.3289 & \\
& 4 & $1,1,1,1,-1$ & 5 & -0.1207 & \\
4 & 2 & $1,2,1$ & 6 & -0.0889 & \\
& 3 & $1,1,1,1$ & 4 & -0.2429 & \\
& 4 & $1,1,1,1$ & 4 & -0.3889 & -0.0617 \\
& 5 & $1,1,1,1,1,-1$ & 6 & -0.2410 & \\
& 6 & $1,1,1,1,1,1,-2$ & 10 & -0.0749 & \\
5 & 2 & $1,3,1$ & 11 & -0.0662 & \\
& 3 & $1,1,1,2$ & 7 & -0.1908 & \\
& 4 & $1,1,1,1,1$ & 5 & -0.3151 & -0.0369 \\
& 5 & $1,1,1,1,1$ & 5 & -0.4224 & -0.1643 \\
& 6 & $1,1,1,1,1,1,-1$ & 7 & -0.3145 & -0.0272 \\
& 7 & $1,1,1,1,1,1,1,-2$ & 11 & -0.1868 & \\
& 8 & $1,1,1,1,1,1,1,1,-3$ & 17 & -0.0475 & \\
\hline
\end{tabular}

Table 2: 


\begin{tabular}{|l|l|l|l|l|l|}
\hline $\mathrm{N}$ & $\mathrm{m}$ & mode & $\omega^{2}$ & \multicolumn{3}{l|}{} \\
\hline & & & $\beta=4$ & $\beta=1$ & $\beta=0.25$ \\
\hline 1 & 0 & $\mathrm{a}$ & - & 1.5540 & 0.4538 \\
& & $\mathrm{~b}$ & 1.9097 & 1.5302 & 1.1073 \\
& & $\mathrm{c}$ & 1.9304 & 1.5565 & 1.1298 \\
& 1 & $\mathrm{t}$ & -0.0459 & -0.0187 & -0.0096 \\
2 & 0 & $\mathrm{a}$ & - & 1.0766 & 0.3604 \\
& & $\mathrm{~b}$ & 1.5110 & 1.0476 & 0.6775 \\
& & $\mathrm{c}$ & 1.5713 & 1.0781 & 0.6963 \\
& 1 & $\mathrm{t}$ & -0.0267 & -0.0050 & -0.0001 \\
& 2 & $\mathrm{~s}$ & -0.7654 & -0.0007 & 0.1121 \\
3 & 0 & $\mathrm{a}$ & 1.7085 & 0.8043 & 0.2941 \\
& & $\mathrm{~b}$ & 1.2163 & 0.7856 & 0.4860 \\
& & $\mathrm{c}$ & 1.2720 & 0.8057 & 0.4932 \\
& 1 & $\mathrm{t}$ & -0.0152 & -0.0013 & 0.0016 \\
& 2 & $\mathrm{~s}$ & -0.4059 & -0.0009 & 0.0617 \\
& 3 & $\mathrm{~s}$ & -1.3497 & 0.0023 & 0.1952 \\
\hline
\end{tabular}

Table 3: 
Figure 1.

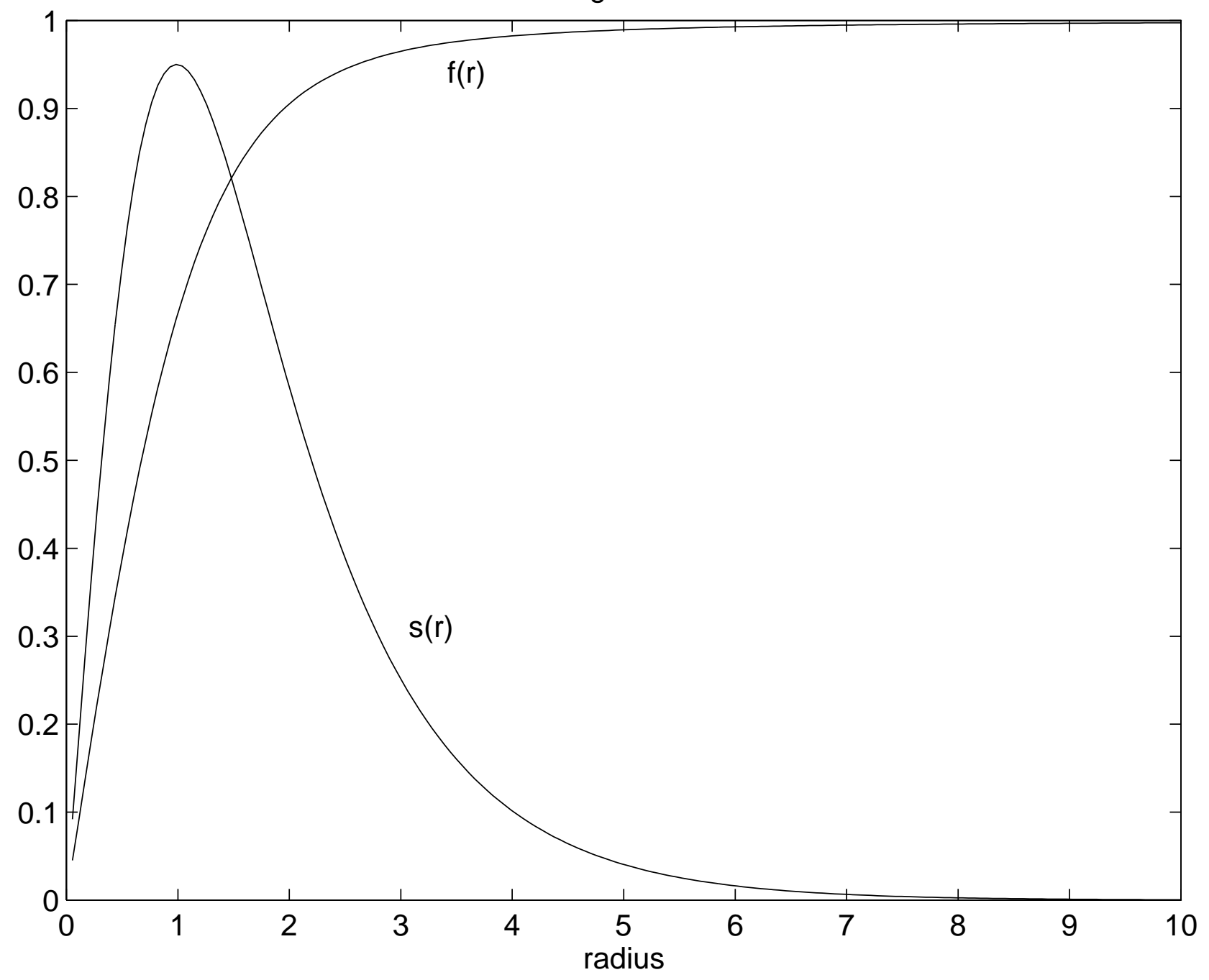


Figure 2.

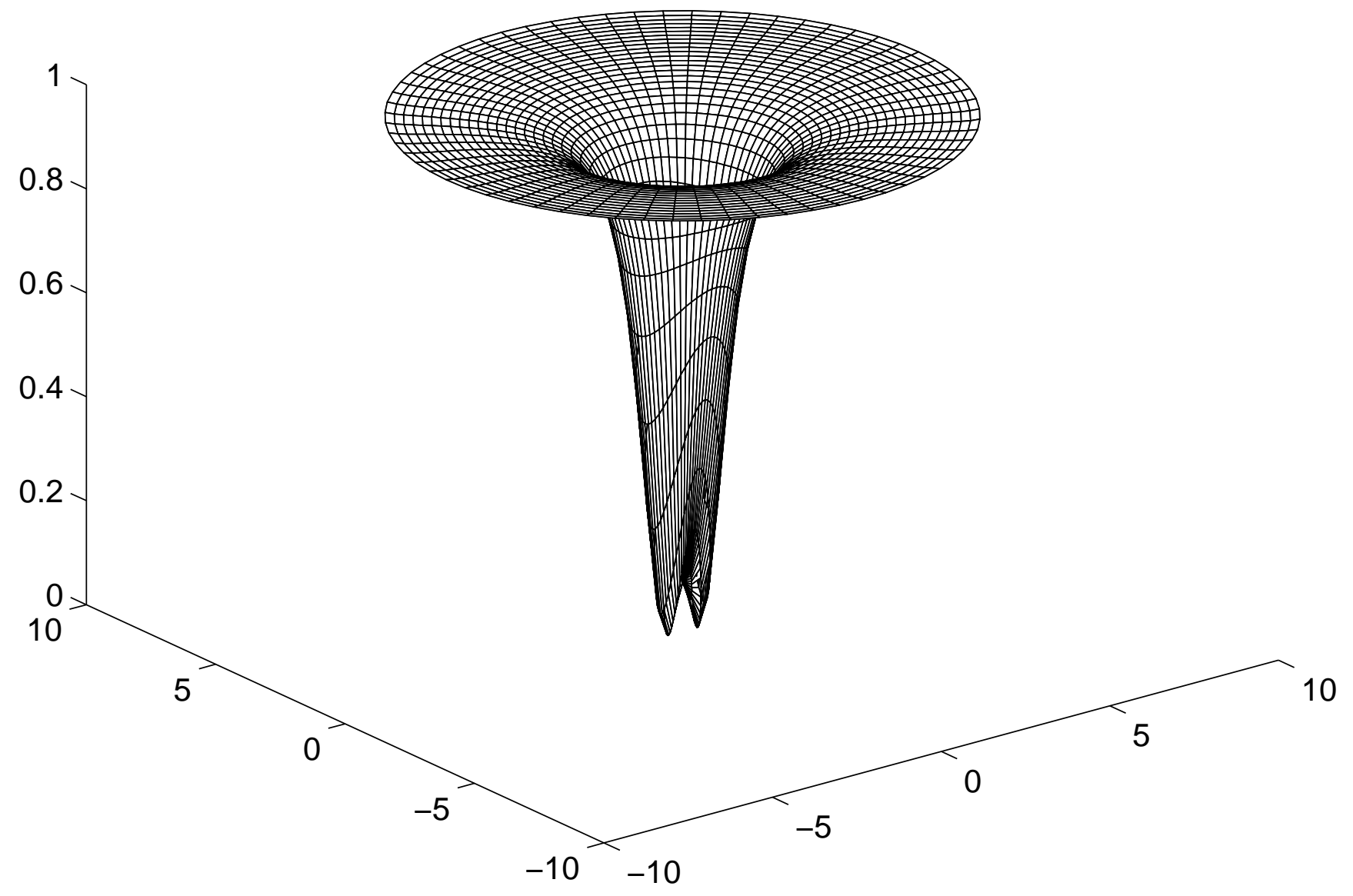


Figure 3.

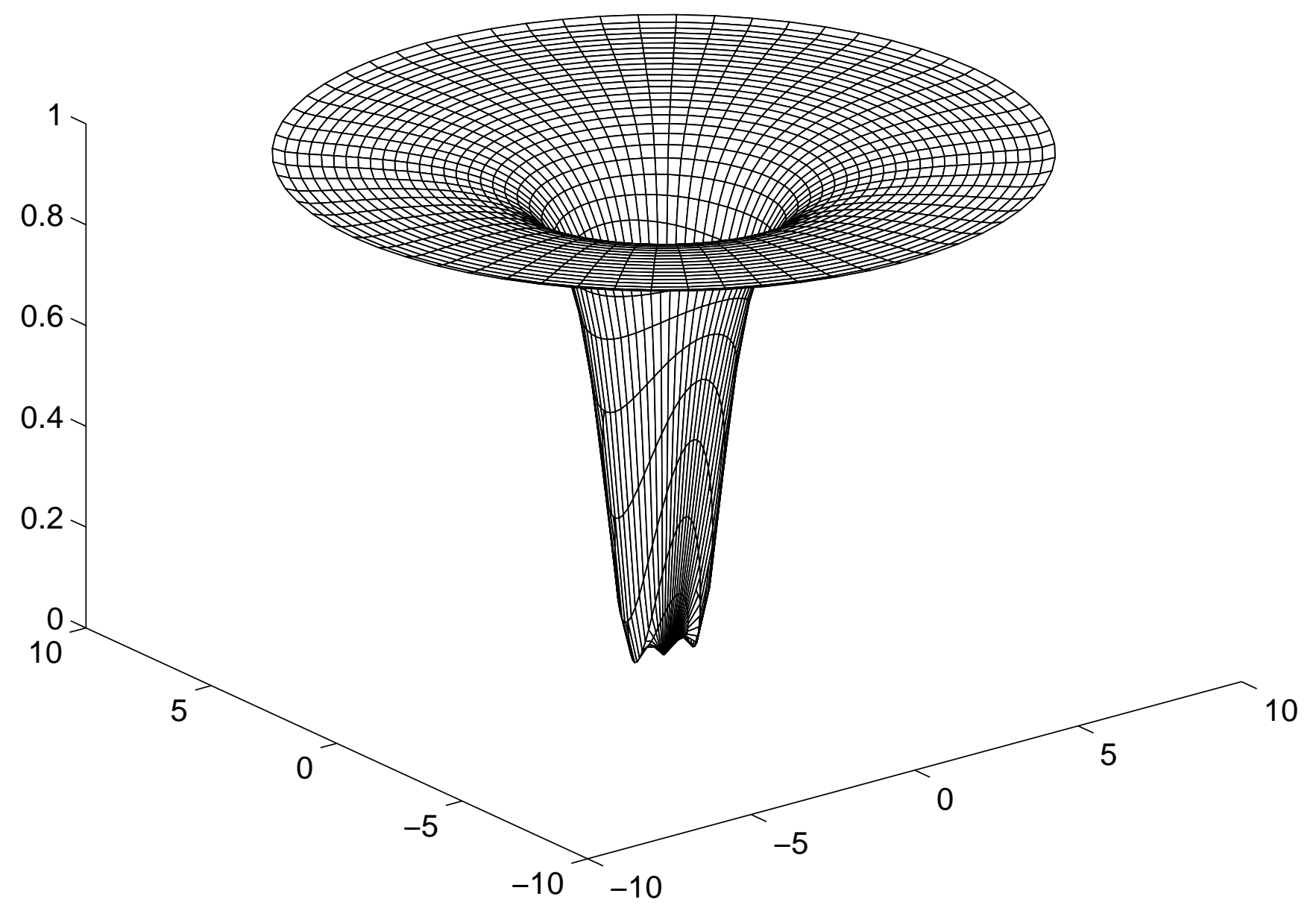


Figure 4.

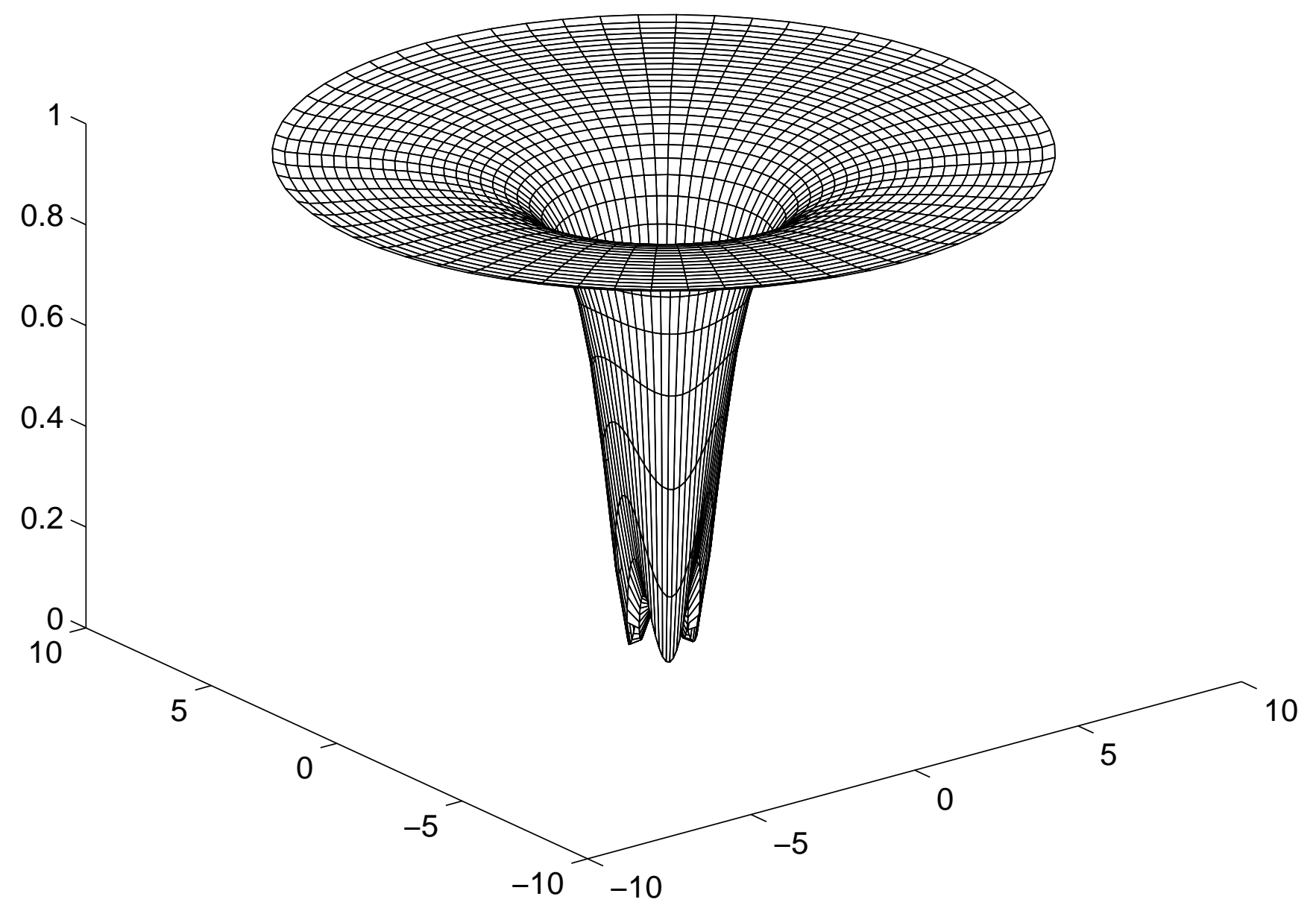


Figure 5.

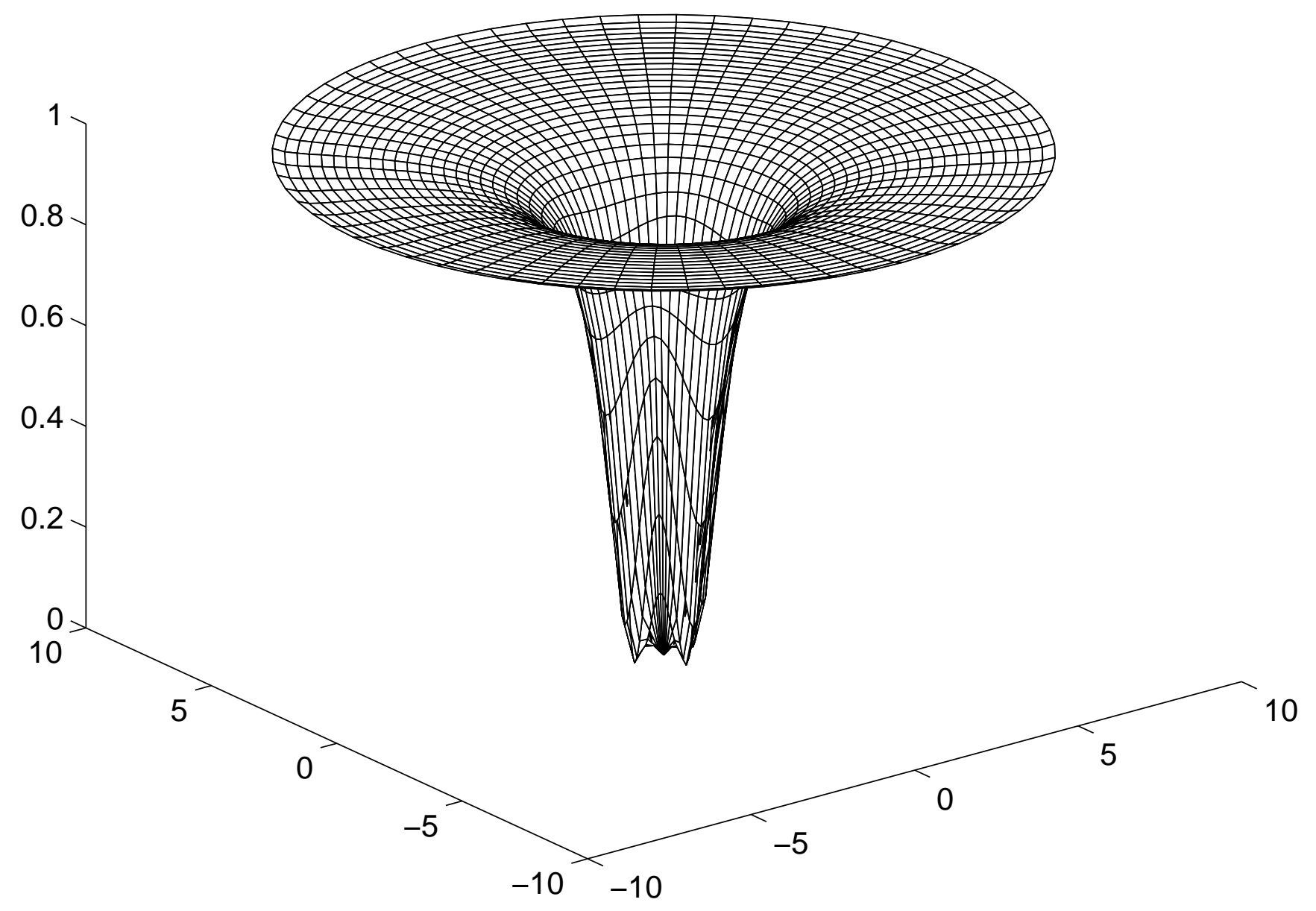


Figure 6

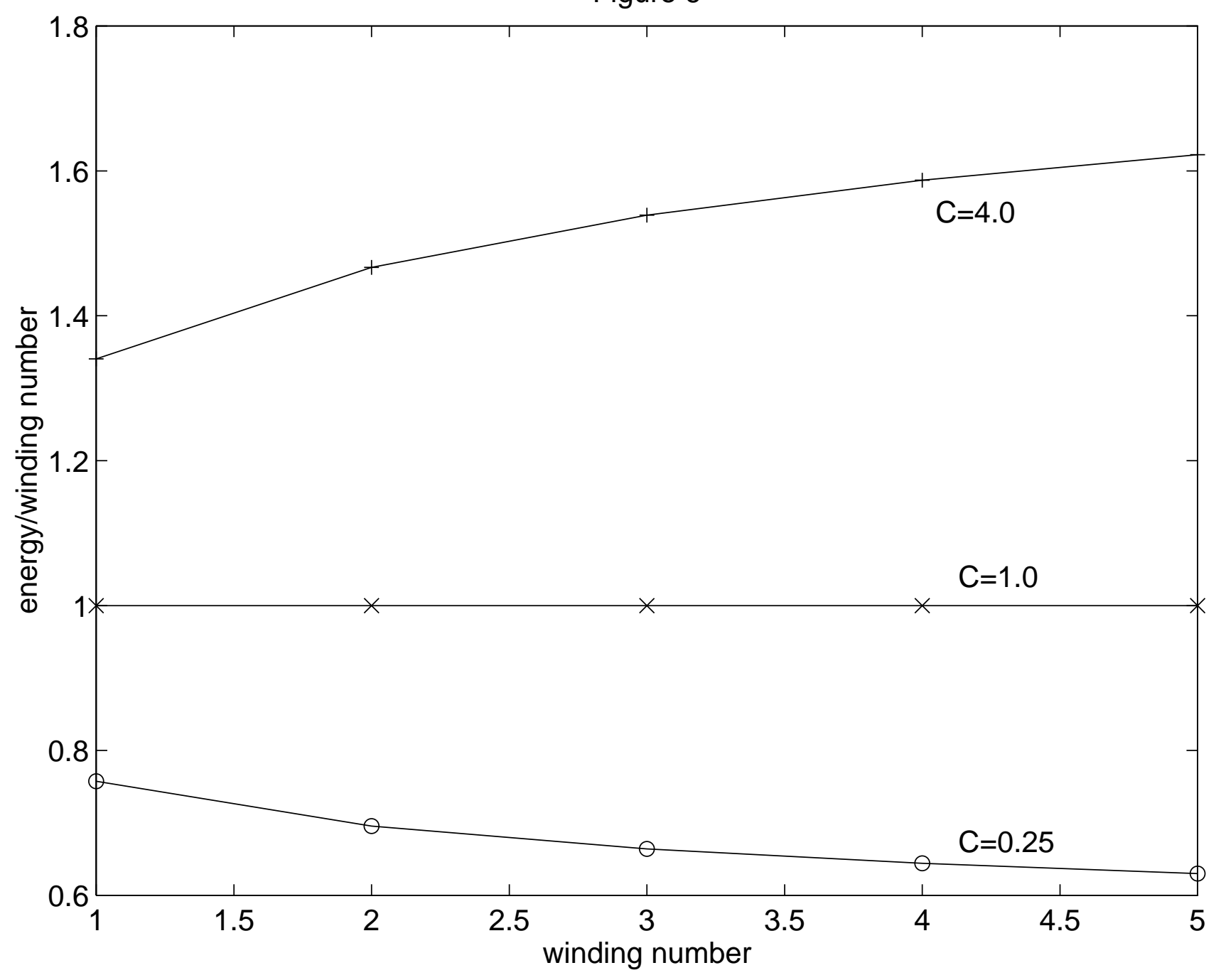

